\title{
Association of fibrous filters for aerosol filtration in predominant Brownian diffusion conditions
}

\author{
Augustin CHARVET ${ }^{1-2}$, Stéphanie PACAULT ${ }^{1-2}$, Soleiman BOURROUS ${ }^{3}$, Dominique THOMAS ${ }^{1-2}$
}

\author{
${ }^{1}$ Université de Lorraine, Laboratoire Réactions et Génie des Procédés (LRGP), UMR 7274, Nancy, F-54000, France. \\ ${ }^{2}$ CNRS, Laboratoire Réactions et Génie des Procédés (LRGP), UMR 7274, Nancy, F-54000, France. \\ ${ }^{3}$ Institut de Radioprotection et de Sureté Nucléaire (IRSN), PSN-RES, SCA, Gif-sur-Yvette, France. \\ Corresponding author: augustin.charvet@univ-lorraine.fr; Tel: +33(0) 372743725
}

\begin{abstract}
$\underline{\text { Abstract }}$
In order to improve the dust retention capacity of air filters and increase their lifetime, the filtration performance of associations of fibrous filters has been investigated. This study highlighted the interest of the addition of a medium or coarse filter, composed of microfibers, upstream of a HEPA filter. Collecting nanostructured particles in the depth of this upstream filter, rather than directly on the surface of a HEPA filter, permits to significantly reduce the pressure drop increase while maintaining a global mass collection efficiency close to the unit. On the one hand, the association of a medium filter and a HEPA filter induces a real energy gain, compared to the implementation of a single HEPA filter. On the other hand, the implementation of a coarse filter, presenting a too low initial collection efficiency, upstream of a HEPA filter leads to mixed results.
\end{abstract}

\section{Keywords}

Nanoparticles, Fibrous filters, Multilayers, Pressure drop

\section{Introduction}

At this time, because of their very high-efficiency, the fibrous filters are the most commonly dedusting systems used for individual and collective protection. High efficiency particulate air (HEPA) filters are used in many applications: containment in medical, pharmaceutical or nuclear facilities, cabin air treatment in automobiles and aircrafts, vacuum and so on. In addition to their high efficiency, these filters, usually non regenerable, may have an important lifetime (i.e. high dust retention capacity) to avoid too frequent replacements.

The performances of these filters are typically measured by the figure of merit (also called quality factor) which represents the ratio of the penetration of particles to the filter pressure drop [1]. A high quality filters are thus characterized by high values of quality factor (i.e. high filtration efficiency and low pressure drop). To further improve the filter performances, a possible solution is to fabricate fibers with diameter lower than $0.5 \mu \mathrm{m}$ (called nanofibers). Nanofiber filters, generally produced by electrospinning [2], have a small fiber size so that they integrate the advantage of a small pore size as well as large surface collection area. Besides, low basis weight also make nanofiber filtration a promising application [3]. As the collection efficiency is a decreasing function of the collector size, nanofiber filters could generally achieve a high collection efficiency. However, the pressure drop across nanofiber filters is higher than across microfiber filters (all other things being equal) even in the slip regime. Wang et al. [4] compared the performances of nanofiber filters and conventional fiber glass filters and highlighted that nanofiber filters have a better figure of merit for particles larger than $100 \mathrm{~nm}$ and a similar one for particles smaller than $100 \mathrm{~nm}$. Furthermore, the authors observed an optimal nanofiber packing density at which the figure of merit is maximal. The characteristics of the nanofiber filters (packing density, fiber diameter) may thus be adjusted according to the size of the particles mostly present in the gaseous effluent.

In practical applications, a thin nanofiber layer is usually placed upstream because of its high filtration efficiency while a downstream microfiber layer provides mechanical strenght. Wang et al. [5] studied the initial filtration performance of dual-layers filters composed of monodisperse micrometer fibers and nanofibers. The theoretical calculation of the quality factor for various layer properties (packing density, thickness, fiber diameter) highlighted that an increase of the thickness and the packing density in the nanofiber layer leads to better performances for particles larger than $100 \mathrm{~nm}$ and to opposite results for particles smaller than $100 \mathrm{~nm}$. Furthermore, increasing the nanofiber diameter leads to better performances for particles smaller than $100 \mathrm{~nm}$ and to worse ones for particles larger than $100 \mathrm{~nm}$; increasing the microfiber diameter induces exactly opposite results. However, initial filtration performances are not sufficient to conclude on the best properties of a filter. It is absolutely necessary to investigate the influence of these layer properties under loading with 
particles. Within this scope, Przekop and Gradon [6] developed a model estimating the temporal performances of bilayer filters consisting of nanofibers in the front layer and microfibers in the rear layer. The highest quality factor was obtained for the smallest nanofibers and microfibers tested and decreased during filter loading. The authors also highlighted that a uniform distribution of nanofibers in the front layer leads to a better quality factor during particle loading. Indeed, in case of thickness heterogeneities, the air flow tends to pass through the thinner (and less resistant) part of the nanofiber layer leading to a decrease of filtration performance. As a means for obtaining a thickness homogeneity of nanofiber mats, Zhang et al. [3] recommend the use of multiple thin layers of nanofibers.

Overall, the solution consisting of adding a thin layer of nanofibers allows increasing the collection efficiency but not countering the pressure drop increase during particle loading and consequently the energy consumption. A possible solution to reduce the pressure drop increase is a better distribution of particles collected within the fibrous filter. Leung et al. [7] performed experimental loading tests with $\mathrm{NaCl}$ submicron particles to evaluate the temporal performances of filters composed of two layers; one with nanofibers and another with microfibers. They notably highlighted that placing the microfiber layer upstream leads to more homogeneous deposit in the filter thickness and consequently to a lower pressure drop increase rate. In the specific case of nanoparticle filtration, the morphology and the size of these particles induce, for the same area density of collected particles, a faster increase of the pressure drop during the filter clogging than for submicronic and micronic particles [8]. This further reduces the lifetime of the fibrous filter and makes even more important the optimization of its retention capacity.

As nanofibers may present a health risk [9-10] and potentially a social acceptability risk, our approach is quite different and consists in a series association of microfiber filters with various properties (packing density, mean fiber diameter).

\section{Materials and Methods}

The fibrous filter or the association in series of fibrous filters were placed downstream of a spark generator (GFG1000, Palas) producing graphite nanostructured particles. Their number size distribution was measured by a SMPS (Scanning Mobility Particle Sizer, nanoDMA 3085 TSI + CPC 3776 TSI). The particle size distribution presents a number median diameter (expressed in electrical mobility-equivalent diameter) of $60 \mathrm{~nm}$ and a Geometric Standard Deviation (GSD) of 1.6. The total number concentration was close to $4.510^{7} \mathrm{~cm}^{3}$. Control valve allows to check the filtration airflow rate whose acquisition is insured by a flow sensor placed downstream of a safety filter.

The characteristics of the fibrous filters are summarized in the table 1 . The analysis of scanning electron microscope (SEM) pictures, performed on about 300 fibers (per filter) permits to obtain the mean fiber diameters. The thickness and packing density of each filter have been determined by two methods:

- A laser trigonometry method (LTM), inspired by the study of Altmann and Ripperger [11], allows obtaining the filter thickness by the measurement of the displacement of a laser between two pictures. For more details, readers may refer to Ribeyre et al. [12]. The packing density may be deduced from this thickness and the weighing of a filter with a known surface.

- A impregnation method (IM), inspired by the method of Schmidt [13], followed by an analysis of SEM pictures allows a local measurement of the properties of filters embedded in an epoxy matrix. A numerical analysis tool have been developed to analyze SEM pictures of the embedded filters. After a binarization and a noise removal process based on filtration operations, the ratio of black and white pixel has been plotted along the filter thickness. The thickness and the mean packing density have been extracted from this profile. For more details on the filter preparation and properties measurement, readers may refer to Bourrous and al. [14].

The two methods lead to similar results except for the thickness measurement of the filter E. This difference may be explained by a loose fiber network and a possible reflection of the laser on the polyethylene terephthalate fibers.

The filters are supported by a grid presenting a negligible collection efficiency and pressure drop. When they are associated in series, the HEPA filter (called filter A) is systematically placed downstream of the medium or coarse one (filter B, C, D or E). The filter pressure drop is continuously recorded during the particle loading. The number particle size distributions are intermittently (every 30 minutes) measured upstream and downstream of the filter (or the series association of filters) using a Scanning Mobility Particle Sizer to obtain the temporal evolution of the fractional number efficiency. 
Table 1: Characteristic properties of tested fiber filters

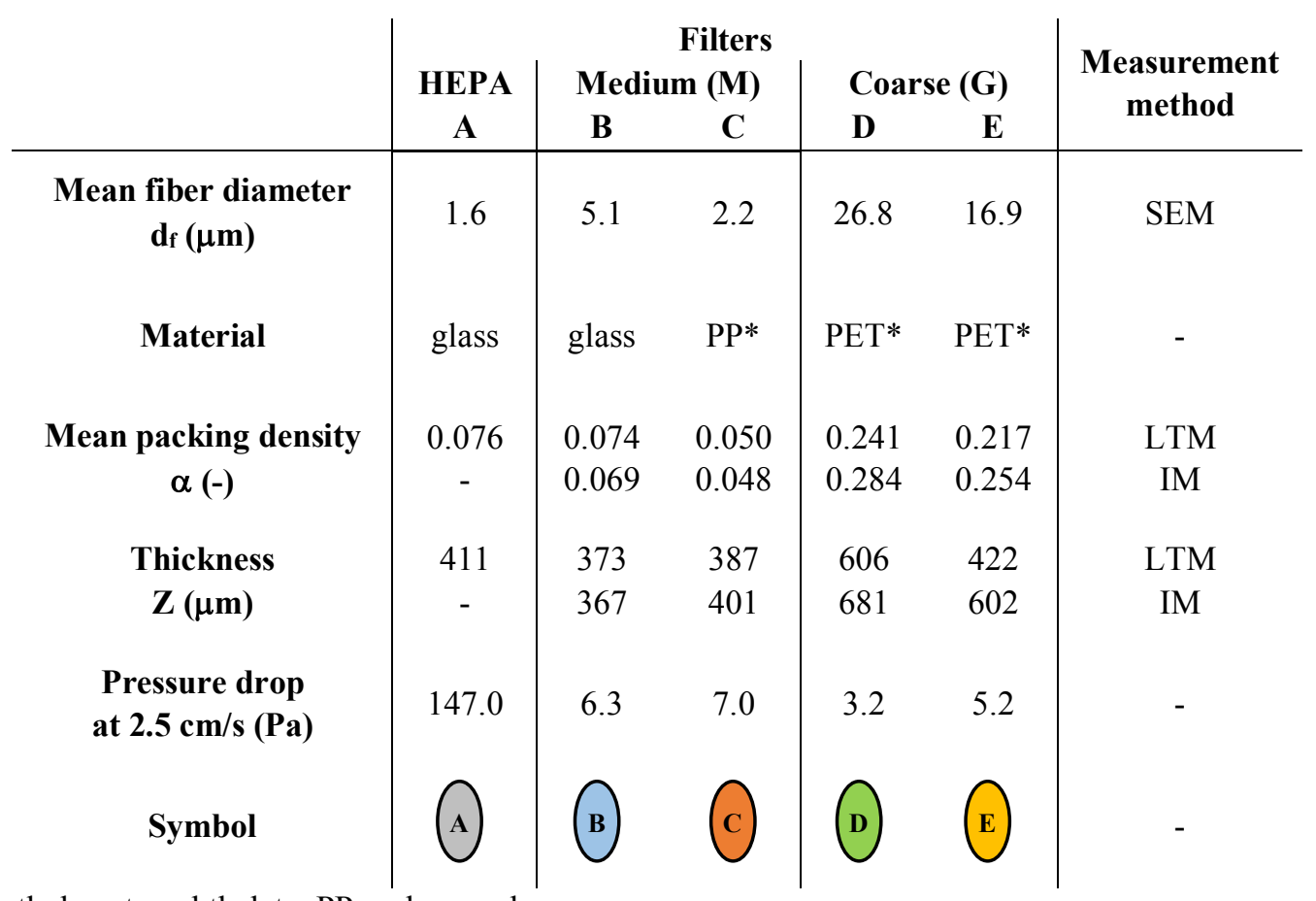

* PET: Polyethylene terephthalate; PP: polypropylene

Upstream and downstream fractional number concentration $\left(\mathrm{C}_{\mathrm{n}, \mathrm{i}}\right)$ may be converted into fractional mass concentrations $\left(\mathrm{C}_{\mathrm{m}, \mathrm{i}}\right)$ according to equation 1 .

$$
\mathrm{C}_{\mathrm{m}, \mathrm{i}}=\frac{\pi \cdot \rho_{\mathrm{P}}}{6} \cdot \mathrm{C}_{\mathrm{n}, \mathrm{i}} \cdot \mathrm{d}_{\mathrm{V}, \mathrm{i}}^{3}
$$

where $\rho_{p}$ is the density of the raw particle material, $d_{v, i}$ the volume equivalent diameter and $C_{n, i}$ the measured fractional number concentration.

As graphite airborne particles are agglomerated and not spherical, the volume equivalent diameter differs from the electric mobility equivalent diameter (with is measured by the SMPS). The effective density, defined as the ratio of aggregate mass to equivalent mobility volume, allows converting the volume equivalent diameter into an electric mobility equivalent diameter and consequently calculating the fractional mass concentrations (cf. equation 2). An experimental protocol, described in details in Charvet et al. [15], provides the relationship between the effective density of the graphite agglomerates and the electric mobility equivalent diameter (cf. equation 3 ).

$$
\begin{aligned}
& C_{m, i}=\frac{\pi \cdot \rho_{e, i}}{6} \cdot C_{n, i} \cdot d_{m, i}^{3} \\
& \rho_{e, i}=20.135 \cdot d_{m, i}^{-1.02}
\end{aligned}
$$

where $\rho_{\mathrm{e}}$ is the effective density of the graphite agglomerates and $\mathrm{d}_{\mathrm{m}, \mathrm{i}}$ the electric mobility equivalent diameter. It should be noted that in the equation 3, the mobility equivalent diameter is expressed in $\mathrm{nm}$ and the effective density in $\mathrm{g} / \mathrm{cm}^{3}$. It was therefore possible to obtain the total mass collected per unit of filter area $\left(\mathrm{m}_{\mathrm{c}, \mathrm{t}}\right)$ during a set period of time $(\Delta \mathrm{t})$ :

$$
\begin{gathered}
m_{c, t}=\frac{Q_{V} \cdot \Delta \mathrm{t}}{\Omega} \cdot \sum_{d_{m, i}}\left(\overline{\mathrm{C}_{\mathrm{m}, \mathrm{i}, \mathrm{up}}} \cdot E_{m, i, t}\right) \\
E_{m, i, t}=\frac{\mathrm{C}_{\mathrm{m}, \mathrm{i}, \mathrm{up}}-\mathrm{C}_{\mathrm{m}, \mathrm{i}, \mathrm{down}}}{\mathrm{C}_{\mathrm{m}, \mathrm{i}, \mathrm{up}}}
\end{gathered}
$$

where $Q_{v}$ is the volumetric air flow rate and $\Omega$ the filter area. $C_{m, i, u p}$ corresponds to the average of the upstream fractional mass concentrations (measured intermittently) and $\mathrm{E}_{\mathrm{m}, \mathrm{i}, \mathrm{t}}$ to the fractional mass efficiency. As particle size distributions are measured every 30 minutes, the temporal evolution of fractional mass efficiency has been fitted by a mathematical law to calculate the total mass collected per unit of filter area at each time. 


\section{Results and Discussion}

\section{Single filter configuration}

Particle loadings have been performed for the five tested filters. The figure 1 represents the temporal evolution of the filter performances. The left chart represents the evolution of the global mass efficiency during particle loading. The right chart is expressed in terms of filter flow resistance and not pressure drop to allow a comparison of experiments realized at various filtration velocities. The flow resistance $(\mathrm{R})$ is defined as the ratio of the filter pressure drop $(\Delta \mathrm{P})$ and the product of the filtration velocity $(\mathrm{U})$ and the gas dynamic viscosity $(\eta)$. This resistance corresponds to the ratio of the filter thickness $(\mathrm{Z})$ and its permeability (B).

$$
\mathrm{R}=\frac{\Delta \mathrm{P}}{\mathrm{U} \cdot \eta}=\frac{\mathrm{Z}}{\mathrm{B}}
$$

For medium and coarse filters, the flow resistance evolution, based on the mass of collected particles, can be divided into three phases. At the beginning of the particle loading, the collection of the particles within the filter induces a slight increase of the pressure drop; this phase is called depth filtration [8]. The transition phase is characterized by an exponential change in the pressure drop due to the appearance of a particle deposit on the filter surface. During this phase, some particles are still collected in the filter depth.

Finally, during the surface filtration phase, particles are collected by the surface deposit and the pressure drop evolution becomes linear due to an increase of the deposit thickness. This underlines that the cake packing density remains constant during the particle loading and consequently that there is no cake compression. For filters B and C, having similar properties, flow resistance evolutions are almost similar and slower at higher velocity. Concerning the filters D and E, which present thicker fibers, a slower increase of the pressure drop is observed.
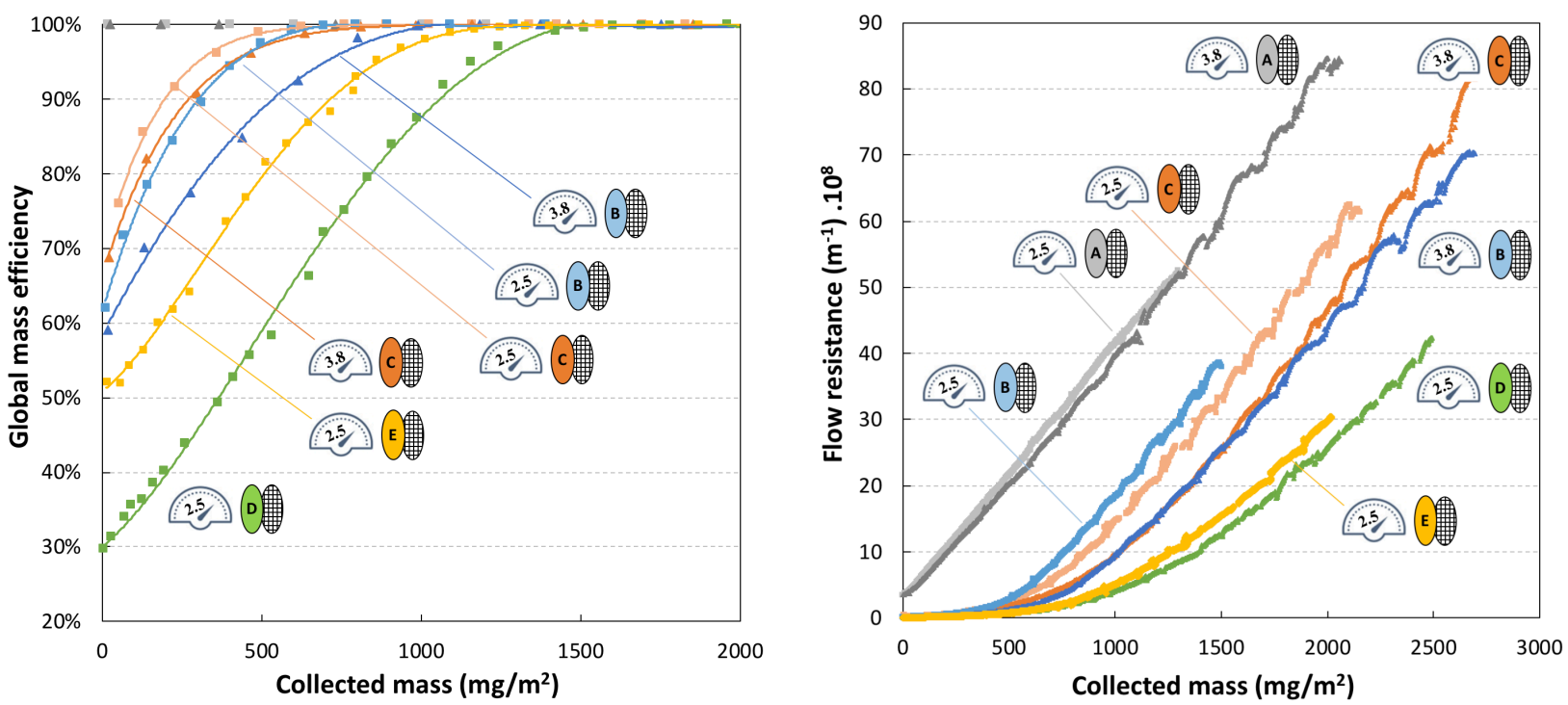

Figure 1: Evolution of the filter performances as a function of collected mass and filtration velocity (the speedometer indicates the filtration velocity in $\mathrm{cm} . \mathrm{s}^{-1}$ )

These various shapes are mainly correlated to collection efficiency. Indeed, the depth filtration phase will be longer for a filter presenting a low initial efficiency and consequently, for a given collected mass, the particles will be deeply located within the filter and the pressure drop lower. Moreover, this homogeneous deposition in the filter depth is emphasized at high velocity as in predominant Brownian diffusion conditions, a velocity increase induces an efficiency decrease.

The filter A, having an efficiency higher than $99.9 \%$ whatever the particle size and the filtration velocity, presents a linear evolution of the flow resistance with time. Indeed, for the HEPA filter, the in-depth filtration phase is reduced to a minimum and the increase of the pressure drop is proportional to the thickness of the particle deposit on the filter surface. The similar flow resistance evolutions of the filter A at 2.5 and $3.8 \mathrm{~cm} . \mathrm{s}^{-1}$ confirm that for a HEPA filter the particle deposition mainly occurs on the surface. Moreover, as the curve slopes during the surface filtration phase are equivalent for each experiment, the characteristics of the cake (notably in terms of porosity) may be considered as quite similar whatever the filtration velocity (in the tested velocity range) and whatever the filter properties. Indeed, according to the diameter of the particle generated, the cake porosity will be close to $97.4 \%$ and $97.2 \%$, for a filtration velocity of 2.5 and $3.8 \mathrm{~cm} . \mathrm{s}^{-1}$, respectively [16]. 
To delay the appearance of the surface filtration phase and promote the depth filtration, a medium or a coarse filter was placed upstream of the HEPA filter during particle loadings. To compare the different associations, results were expressed in terms of filter flow resistance; this parameter was preferred to an estimation of the quality factor because of a collection efficiency higher than $99.9 \%$ for all the tested associations (cf. figure 2). The resistance evolutions of the HEPA filter, at various filtration velocities, have been added on the figure 2 in order to highlight the contribution of a medium or coarse filter addition.
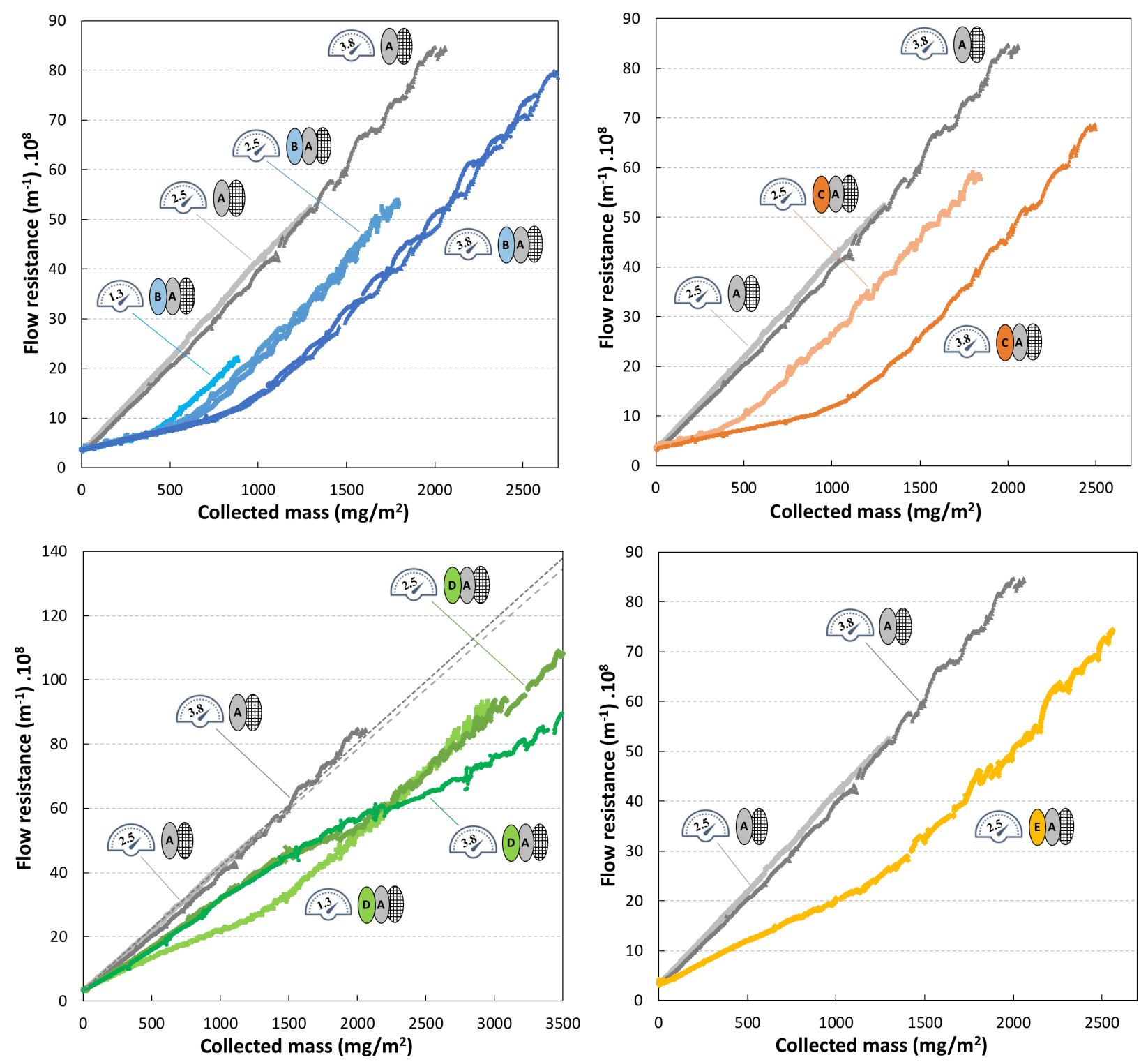

Figure 2: Evolution of the filter flow resistance as a function of collected mass for various filter series associations

As upstream filters present low initial pressure drops, the initial pressure drop of series associations is of the same order of magnitude as that of the HEPA filter. This observation is particularly interesting because it means that the implementation of a medium or coarse filter does not induce an additional operational cost. The results tend to validate our approach as the resistance evolution of the filter associations is much slower than that of the HEPA filter alone. Indeed, the slightest efficiency of the medium or coarse filters induces a penetration of particles in the filter depth, a better repartition of the particle mass in the filter volume and thus a delay of the linear increase of pressure drop for the associations of filters. As, in the case of nanoparticles, the collection is mainly governed by Brownian diffusion, this phenomenon is all the more emphasized as the filtration velocity is important. A second set of measurements realized on the association $(\mathrm{B}+\mathrm{A})$ at 2.5 and $3.8 \mathrm{~cm} \cdot \mathrm{s}^{-1}$ highlights a high data reproducibility. 
If associating two filters in series seems relevant using medium filter (B or C), the association of a HEPA filter and a coarse filter (D or E), i.e. filters having a too low initial efficiency, seems to give mixed results. Indeed, such a penetration induces a quicker increase of the pressure drop because of a cake formation at the surface of the HEPA filter. Furthermore, if working at more important filtration velocities allows a better homogenization of the deposit in the depth of the filter B or $\mathrm{C}$, for the filters with low initial efficiency (filters D and E), the influence of the filtration velocity is more complex to understand. Indeed, according to the value of the filtration velocity, the particle collection can occur in the depth or in the surface of the filter. A low velocity (and consequently a higher collection efficiency) will first induce a collection of particles in the depth of the coarse filter and then the formation of a cake at the coarse filter surface. The shape of the resistance evolution of associations $(\mathrm{D}+\mathrm{A})$ and $(\mathrm{E}+\mathrm{A})$ is thus similar to that of the associations $(\mathrm{B}+\mathrm{A})$ and $(\mathrm{C}+\mathrm{A})$, even if the kinetics is quicker because of a too low initial efficiency and consequently a collection of particles occurring also on the surface of the HEPA filter. On the other hand, at higher velocity, the efficiency of the coarse filter decreases strongly and leads to a collection of particles, at first, mainly on the surface of the HEPA filter. This cake formation causes an important increase of the pressure drop at the beginning of clogging. Afterward, because of the increase of the efficiency of the coarse filter (due to particle accumulation), particles will be mainly collected in its depth and finally on the surface.

To verify these hypotheses, an experiment was carried out either with contiguous filters tested in the same filter holder but with each filter in a filter holder. Both filter holders (separated by approximatively 50-60 cm) are associated in series, the first containing the coarse filter (filter D in this experiment) and the second the HEPA filter (filter A). The figure 3 (left) highlights that the evolution of global pressure drop of the association $(\mathrm{D}+\mathrm{A})$ is identical whatever the tested configuration (i.e. contiguous filters or placed in two filter holders). This result reproducibility for the two tested configurations was also verified for the association $(B+A)$. Furthermore, in the separated filters configuration, the temporal resistance evolution of each filter allows to validate our previous hypothesis. Indeed, at first, the performances of the filter association seem to be mainly controlled by the HEPA filter, on the surface of which the cake formation leads to a linear increase of pressure drop. This is confirmed by the figure 3 (right) highlighting that a majority of the particle mass is collected by the HEPA filter at the beginning of the particle loading. Subsequently, the increase of the efficiency of the coarse filter induces a slowing down of the pressure drop increase. Finally, when the coarse filter reaches a global mass efficiency close to the unity, the formation of a cake at its surface leads to the reappearance of linear increase of pressure drop.
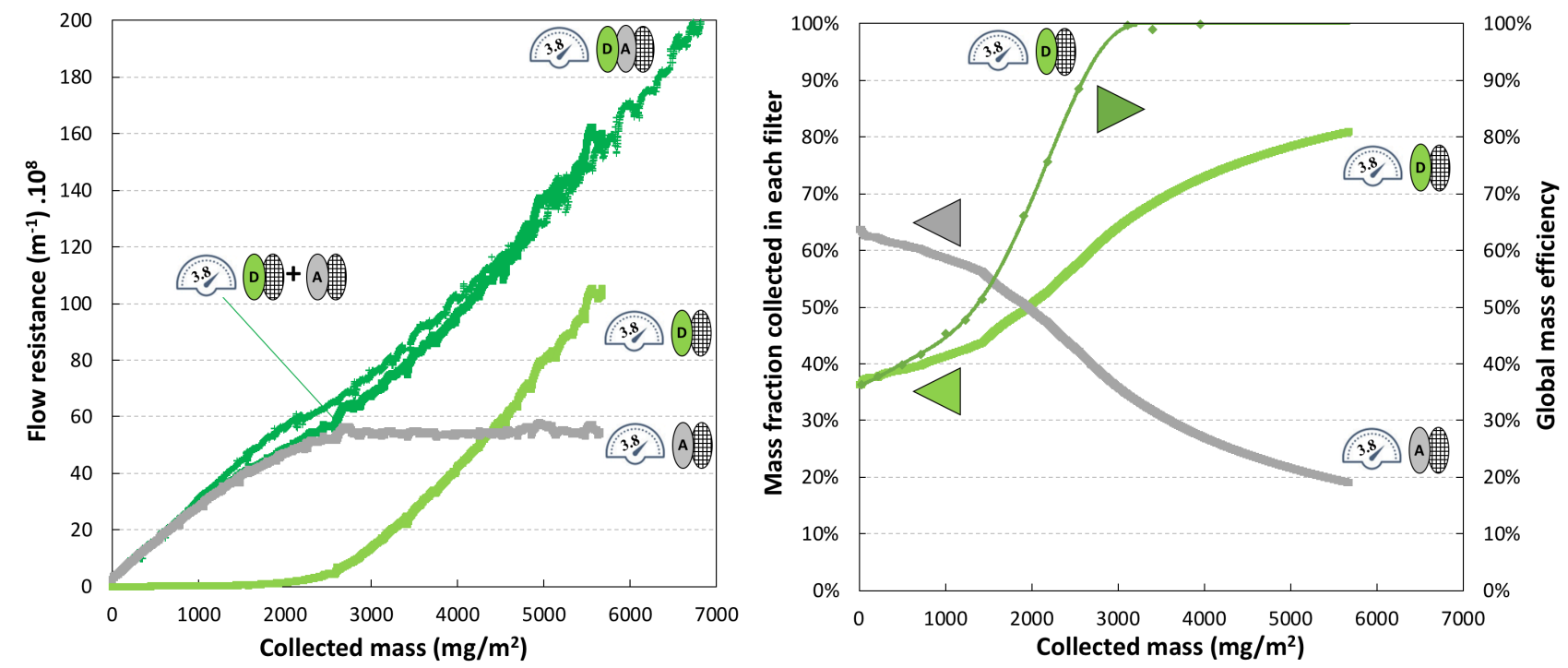

Figure 3: Resistance evolutions of the ( $D+A)$ association according to the configuration (contiguous or separated) (left); Collection efficiency and mass fraction collected in each filter in the separated configuration (right)

A slight overestimation of the pressure drop can be observed for the contiguous configuration. Some possible explanations are particle diffusion losses in the ducts between the two filter holders (for the separated configuration) and/or interactions particles/fibers due to the formation of a particle cake at the surface of the HEPA filter and thus partially in the depth of the coarse filter (for the contiguous configuration). Besides, the filter manufacturing process may induce some heterogeneities between two identical filters. 


\section{Series association configuration $(M+M)$}

Given the good performances of the associations $(\mathrm{B}+\mathrm{A})$ and $(\mathrm{C}+\mathrm{A})$ at a $3.8 \mathrm{~cm} \cdot \mathrm{s}^{-1}$ filtration velocity, a series of experiments was performed at this same velocity by associating in series two medium filters, $(B+B)$ on one hand and $(C+C)$ on the other hand (cf. figure 4). By comparison, the evolutions of the resistance of associations $(\mathrm{B}+\mathrm{A})$ and $(\mathrm{C}+\mathrm{A})$ are also represented.

The initial pressure drop of medium filters being very low (compared with the one of HEPA filter), the series associations of medium filters present a low resistance at the beginning of the particle loading. Afterward, when the global mass efficiency becomes close to the unit and when a cake forms on the surface of the first filter, the increase of the pressure drop becomes linear and the slope of the resistance increase becomes similar to the one obtained in the case of an association (CO+HEPA). Nevertheless, in spite of these advantageous temporal evolutions of the pressure drop, the efficiencies of these associations of medium filters at the beginning of the filtration (lower than $90 \%$ ) can turn out to be critical, in particular if these filters are implemented for containment.
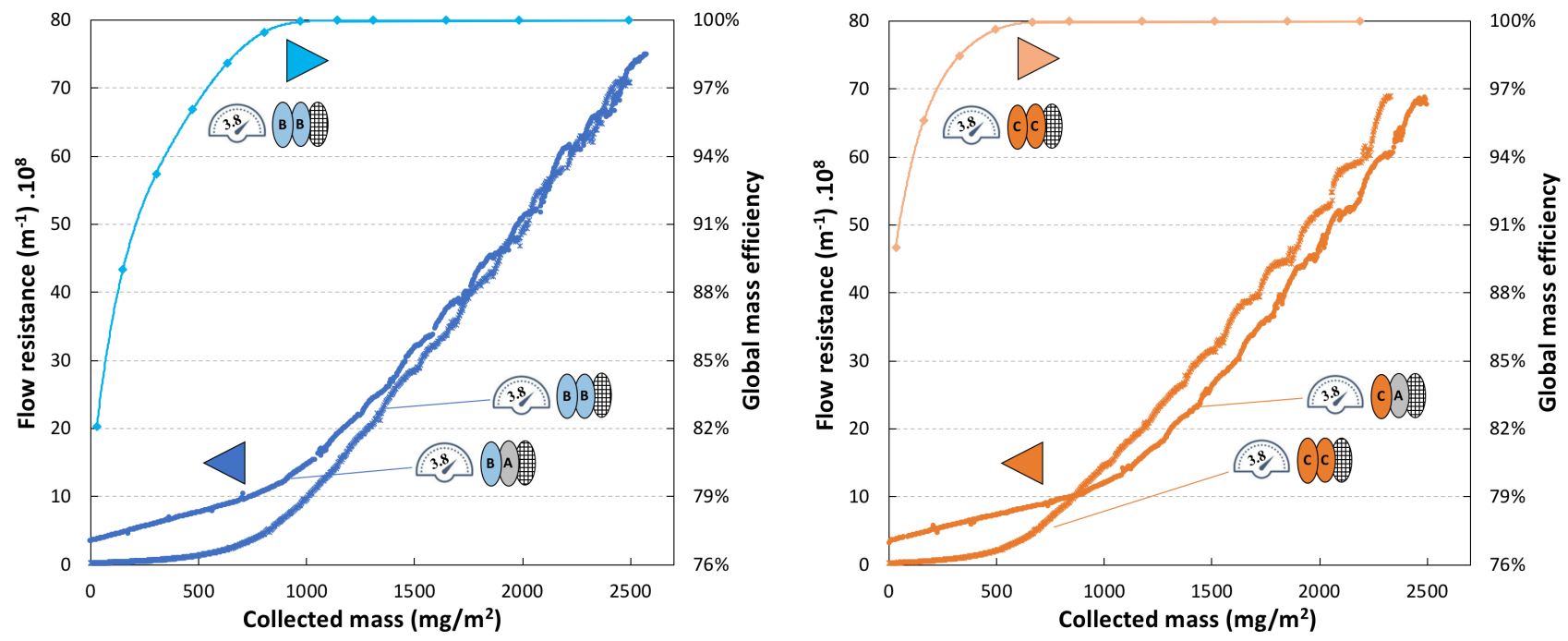

Figure 4: Performance evolution of the coarse filter associations $(C O+C O)$ at a filtration velocity of $3.8 \mathrm{~cm} . \mathrm{s}^{-1}$

\section{Energy cost of associations $(M+H E P A)$ or $(G+H E P A)$}

To quantify the profit related to the addition of a medium or coarse filter upstream of a HEPA filter, the energy cost per mass of collected particles has been determined during each particle loading. This parameter is cumulated over time to obtain the total energy cost (CEC) expanded by each filter or association for the collection of a given particle mass (cf. equation 7).

$$
\mathrm{CEC}=\sum_{t=0}^{t_{f}}\left(\frac{1}{m_{c, t}} \cdot \int_{0}^{t_{f}} \Delta P \cdot t \cdot Q_{V}\right)
$$

As expected, for the filter A, the evolution of the cumulative energy cost is linear during the particle loading (cf. figure 5). Indeed, for a HEPA filter, the pressure drop is proportional to the cake thickness and consequently to the mass collected at the filter surface. This cumulative energy cost evolves more slowly for the filter series associations, notably for $(\mathrm{B}+\mathrm{A})$ and $(\mathrm{C}+\mathrm{A})$ associations. For a given collected mass, the gap between the cumulative energy costs of the filter $\mathrm{A}$ and of a series association represents the energy gain due to the addition of a filter upstream of a HEPA filter. Similarly, for a given cumulative energy cost, the gap between the mass collected per unit area by the filter A and by a series association represents the collected mass gain. Examples of energy and mass gains are given in the table 2, either by setting an objective in terms of collected final mass, or by defining a maximal acceptable energy cost. 

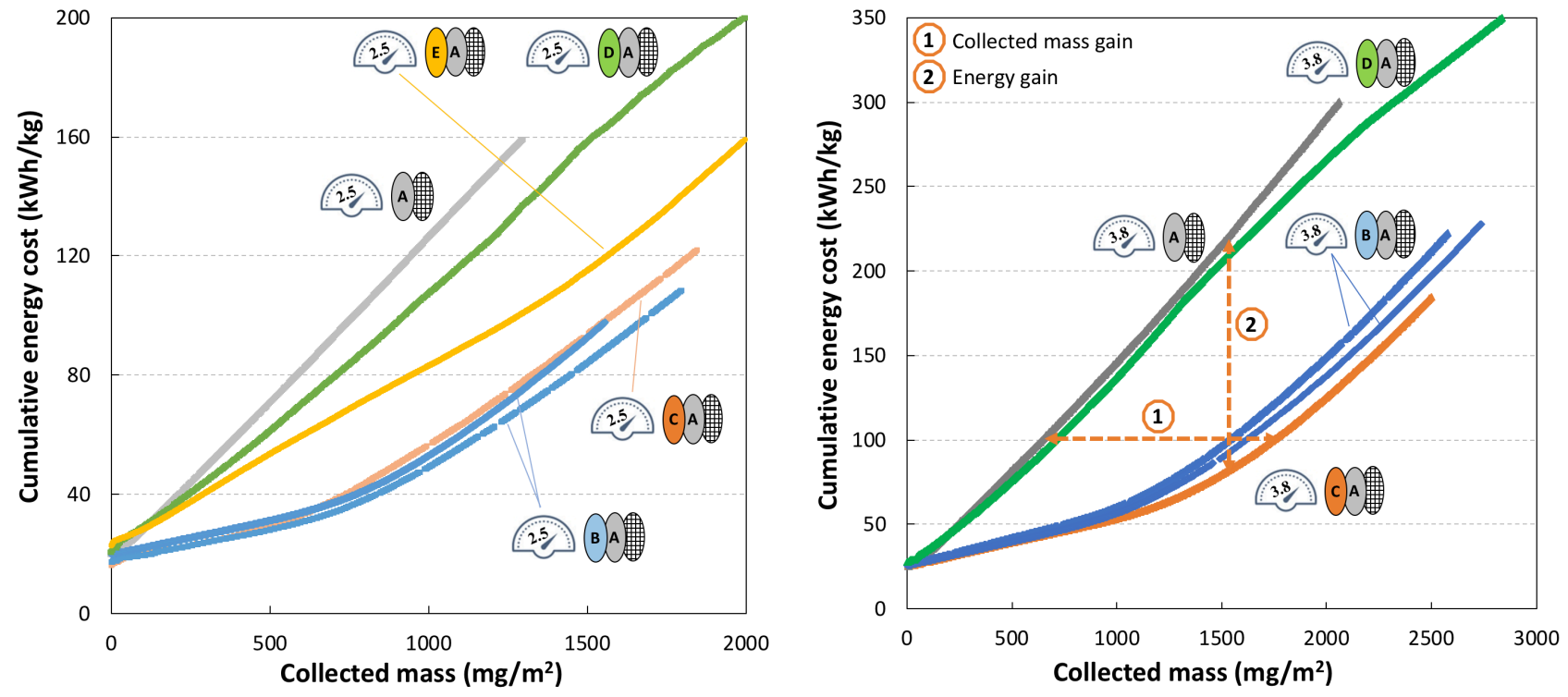

Figure 5: Cumulative energy cost as a function of collected mass for various filter series associations

These gains are all the more important that the filtration velocity is; and decrease if the upstream filter presents a too low initial collection efficiency (as in the case of the coarse filters D and E). Furthermore, after a given time of particle loading (i.e. after an important collected mass per unit area), there is a stabilization of the energy and mass gains. When a cake appears on the surface of the upstream filter, the slope of resistance evolution becomes quite similar that the one of the filter A and consequently the gains become constant. It should be noted that the slope discontinuity for the (D+A) association may be assigned to the transition from a particle collection at the HEPA surface to a particle collection in the coarse filter depth (cf. figure 3).

Table 2: Energy and mass gains of filter series associations

\begin{tabular}{|c|c|c|c|c|c|}
\hline & \multicolumn{4}{|c|}{ Collected mass $\left(\mathrm{mg} / \mathrm{m}^{2}\right)$} \\
\hline & & 500 & 1000 & 1500 & 2000 \\
\hline \multirow{8}{*}{ 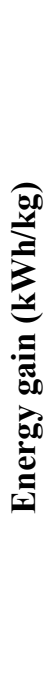 } & \multirow{4}{*}{ 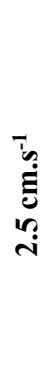 } & $\begin{array}{l}42.5 \\
38.9\end{array}$ & $\begin{array}{l}77.1 \\
72.8\end{array}$ & - & - \\
\hline & & 41.4 & 69.8 & - & - \\
\hline & & 9.5 & 18.9 & - & - \\
\hline & & 17.1 & 43.1 & - & - \\
\hline & \multirow{4}{*}{ 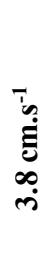 } & 40.2 & 86.2 & 119.3 & 141.9 \\
\hline & & 42.5 & 90.0 & 126.6 & 130.8 \\
\hline & & 49.5 & 99.5 & 137.2 & 155.1 \\
\hline & & 6.0 & 9.1 & 10.4 & 25.2 \\
\hline
\end{tabular}

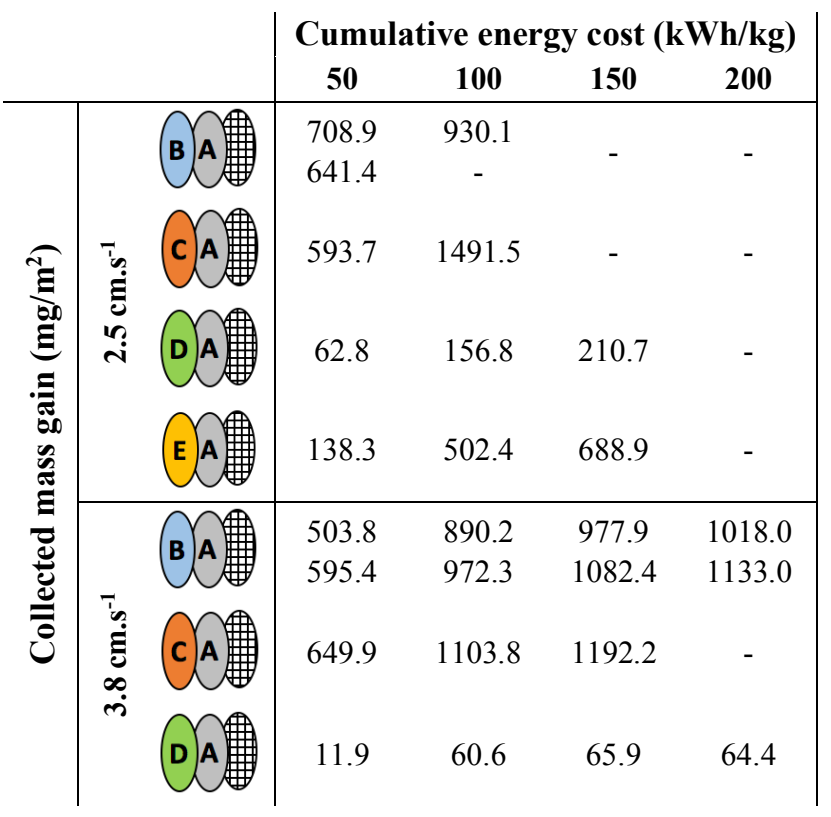




\section{Mass balance}

To validate our calculation approach of the collected mass, a mass balance is carried out at the end of each experiment. The table 3 summarizes the final collected masses calculated from the particle size distributions (cf. equation 4) and the final collected masses coming from weighing at the beginning and the end of each particle loading.

Table 3: Final mass balance for each experiment

\begin{tabular}{|c|c|c|c|c|c|c|c|c|c|c|c|c|c|c|}
\hline \multirow{2}{*}{\multicolumn{2}{|c|}{$\begin{array}{c}\text { Configuration } \\
\text { Filtration velocity }(\mathrm{cm} / \mathrm{s})\end{array}$}} & \multicolumn{2}{|c|}{ A } & \multirow[b]{2}{*}{1.3} & \multirow[b]{2}{*}{2.5} & \multicolumn{2}{|c|}{ BA击曲 } & \multirow[b]{2}{*}{3.8} & \multirow[b]{2}{*}{2.5} & \multirow[b]{2}{*}{3.8} & \multirow[b]{2}{*}{1.3} & \multicolumn{2}{|c|}{ DA } & \multirow[b]{2}{*}{2.5} \\
\hline & & 2.5 & 3.8 & & & 2.5 & 3.8 & & & & & 2.5 & 3.8 & \\
\hline \multirow{3}{*}{$\begin{array}{c}\text { Collected } \\
\text { mass } \\
\left(\mathrm{g} / \mathrm{m}^{2}\right)\end{array}$} & Calculated & 1.30 & 2.06 & 0.89 & 1.80 & 1.56 & 2.58 & 2.74 & 1.85 & 2.50 & 2.99 & 5.55 & 19.98 & 2.56 \\
\hline & Weighed & 1.17 & 1.73 & 0.57 & 1.45 & 1.38 & 2.16 & 2.16 & 1.45 & 2.09 & 2.65 & 5.76 & 20.90 & 2.33 \\
\hline & Relative gap (\%) & 10.2 & 16.0 & 36.3 & 19.4 & 11.3 & 16.3 & 21.2 & 21.6 & 16.5 & 11.3 & 3.8 & 4.6 & 8,9 \\
\hline
\end{tabular}

The deviation between calculated and weighted final collected mass is close to $10-20 \%$ for each experiment. For the calculated mass, the uncertainty is mainly due to the CNC particle concentration accuracy $( \pm 10 \%)$ and to the precision of the relation used to convert number particle concentration into mass particle concentration (cf. equation 3 ). As the collection efficiency is close to $100 \%$ for each filter association, only the uncertainty on the upstream particle concentration shall be taken into account. An accuracy of $\pm 10-15 \%$ may be retained for the total calculated collected mass. For the weighed mass, the precision balance accuracy leads to an uncertainty of about $140 \mathrm{mg} / \mathrm{m}^{2}$. Furthermore, the deposition of particles (by diffusion) on the filter holder and the ducts may explain the quasi-systematic underestimation of the weighed mass, in comparison with the calculated one. Even if the mass balance is not perfect, this does not call into question the previous conclusions as collected mass are calculated with the same approach for each experiment.

\section{Conclusion}

This study highlighted the interest of the addition of a medium or coarse filter (composed of microfibers) upstream of a HEPA filter. Collecting particles in the depth of this upstream filter, rather than directly on the surface of a HEPA filter (standard configuration), permits to significantly reduce the pressure drop increase while maintaining a global mass collection efficiency close to unit. The association of medium and HEPA filters engenders a real energy gain, in comparison with the implementation of a HEPA filter, while the implementation of a coarse filter, presenting a too low initial collection efficiency, leads to mixed results. The research prospects of this study will be to find a medium filter allowing to obtain the highest possible energy gain. For this purpose, the first step should consist in developing a predictive model of the fibrous filter performances, in terms of collection efficiency and pressure drop. This model, under development, should provide the theoretical performances of a large number of fibrous filters with various characteristics in terms of packing density, fiber diameter and thickness. Forward, the final objective will be to find the optimal medium filter characteristics and consequently the optimal association of filters, i.e. the association presenting the lowest possible pressure drop increase.

\section{Acknowledgments}

This work is a part of the LIMA joint research program between the Institut de Radioprotection et de Sureté Nucléaire (French Institute for Radiological Protection and Nuclear Safety) and the Laboratoire Réactions et Génie des Procédés (Reactions and Chemical Engineering Laboratory). 


\section{References}

[1] Brown, R. C. (1993). Air Filtration: An Integrated Approach to the Theory and Applications of Fibrous Filters. Pergamon Press, Oxford, UK.

[2] Barhate, R.S., Ramakrishna, S. (2007). Nanofibrous filtering media: Filtration problems and solutions from tiny materials. Journal of Membrane Science, 296, 1-8.

[3] Zhang, Q., Welch, J., Park, H., Wu, C.Y., Sigmund, W., Marijnissen, J.C.M. (2010). Improvement in nanofiber filtration by multiple thin layers of nanofiber mats. Journal of Aerosol Science, 41, 230-236.

[4] Wang, J., Kim, S.C., Pui, D.Y.H. (2008). Investigation of the figure of merit for filters with a single nanofiber layer on a substrate. Journal of Aerosol Science, 39, 323-334.

[5] Wang, J., Kim, S.C., Pui, D.Y.H. (2008). Figure of Merit of Composite Filters with Micrometer and Nanometer Fibers. Aerosol Science and Technology, 42, 722-728.

[6] Przekop, R., Gradon, L. (2008). Deposition and Filtration of Nanoparticles in the Composites of Nano- and Microsized Fibers. Aerosol Science and Technology, 42, 483-493.

[7] Leung, W.W.F., Hung, C.H., Yuen, P.T. (2009). Experimental Investigation on Continuous Filtration of Sub-Micron Aerosol by Filter Composed of Dual-Layers Including a Nanofiber Layer. Aerosol Science and Technology, 43, 11741183.

[8] Thomas, D., Charvet, A., Bardin-Monnier, N., Appert-Collin, J.C. (2017). Aerosol Filtration. ISTE Press/Elsevier.

[9] Schinwald, A., Murphy, F.A., Prina-Mello, A., Poland, C.A., Byrne, F., Movia, D., Glass, J.R., Dickerson, J.C., Schultz, D.A. Jeffree, C.E., MacNee, W., Donaldson, K. (2012). The Threshold Length for Fiber-Induced Acute Pleural Inflammation: Shedding Light on the Early Events in Asbestos-Induced Mesothelioma. Toxicologial Sciences, 128, 461470.

[10] Wick, P., Clift, M.J.D., Rosslein, M., Rothen-Rutishauser, B. (2011). A brief summary of carbon nanotubes science and technology: A health and safety perspective. ChemSusChem, 4, 905-911.

[11] Altmann, J., Ripperger, S. (1997). Particle deposition and layer formation at the crossflow microfiltration. Journal of Membrane Science, 124, 119-128.

[12] Ribeyre, Q., Charvet, A., Vallières, C., Thomas, D. (2017). Impact of relative humidity on a nanostructured filter cake - experimental and modelling approaches. Chemical Engineering Science, 161, 109-116.

[13] Schmidt, E. (1989). Experimental investigation into the compression of dust cakes deposited on filter media. Powder Technology, 60, 173-177.

[14] Bourrous, S., Bouilloux, L., Ouf, F.X., Appert-Collin, J.C., Thomas, D., Tampère, L., Morele, Y. (2014). Measurement of the nanoparticles distribution in flat and pleated filters during clogging. Aerosol Science and Technology, 48, 392-400.

[15] Charvet, A., Bau, S., Paez Coy, N.-E., Bémer, D., Thomas, D. (2014). Characterizing the effective density and primary particle diameter of airborne nanoparticles produced by spark discharge using mobility and mass measurements (tandem DMA/APM). Journal of Nanoparticle Research, 16:2418.

[16] Thomas, D., Ouf, F.X., Gensdarmes, F., Bourrous, S., Bouilloux, L. (2014). Pressure drop model for nanostructured deposits. Separation and Purification Technology, 138, 144-152. 